\title{
Late adult onset chorea with typical pathology of Hallervorden-Spatz syndrome
}

\author{
D A Grimes, A E Lang, C Bergeron
}

\begin{abstract}
Senile chorea is a well recognised but poorly understood clinical entity characterised by a slowly progressive, generalised chorea in elderly people without mental deterioration or a clear underlying cause. The Hallervorden-Spatz syndrome is typically thought of as a paediatric condition with extrapyramidal features and dementia. However, it has been described in adults usually presenting with parkinsonism plus dementia. An elderly woman with slowly progressive chorea without dementia was found at postmortem to have the pathological features originally described by Hallervorden and Spatz. This association has not previously been reported.

(F Neurol Neurosurg Psychiatry 2000;69:392-395)
\end{abstract}

Keywords: Hallervorden-Spatz syndrome; neurodegeneration with brain iron accumulation type 1 ; senile chorea

The diagnosis of Hallervorden-Spatz syndrome encompasses a considerable range of disorders, all with the core pathological features of iron deposition, axonal spheroids, and gliosis of the pallidum and substantia nigra. ${ }^{12}$ It has recently been reclassified as "neurodegeneration with brain iron accumulation type 1(NBIA-1)" however, most authors continue to use the original terminology. Most cases with this pathology begin in childhood or early adolescence with extrapyramidal dysfunction and dementia, and follow a relentlessly progressive course. ${ }^{1}$ Adult onset cases have been described but typically have prominent dementia associated with parkinsonism..$^{3-5}$ Chorea, although commonly described in paediatric cases, is not found in isolation and has not been reported in adults with the pathological features originally described by Hallervorden and Spatz. We report on a patient with the clinical diagnosis of "senile" or late adult onset chorea who was found to have this pathological diagnosis on postmortem examination.

\section{Case report}

A 76 year old woman was referred with a 1 year history of unsteady gait and unusual movements of her head and upper limbs that had been noticed by her family. Her medical history included myocardial infarction 20 years previously with mild congestive heart failure, peripheral vascular disease, and arthritis. Her medications were digoxin, furosemide, and ibuprofen. There was no known neuroleptic or toxic exposure, no history of Sydenham's chorea or rheumatic fever, and no family history of abnormal movements.

Her initial mental status examination showed an alert, fully oriented woman with only mild difficulty in tasks of attention. Her motor examination was normal except for the mild generalised choreic movements. The rest of her neurological examination, including extraocular movements, was normal except for slight unsteadiness when walking and mild difficulty with tandem gait.

Laboratory investigations including complete blood count, blood smear, thyroid studies, liver function tests, erythrocyte sedimentation rate, ceruloplasmin, anticardiolipin antibodies, antinuclear antibody, and rheumatoid factor were normal. Huntington's disease molecular genetic testing for the CAG trinucleotide repeat expansion was negative (number of repeats $=23$ and 21). Neuropsychological testing disclosed only mild decline in cognitive functioning and was considered not to be consistent with any significant degree of dementia. Head MRI, done 2 years after the onset of her chorea, demonstrated decreased signal intensity in the putamen, caudate, substantia nigra, and dentate nuclei bilaterally (fig 1). There were also multiple small non-specific focal white matter lesions seen in the centrum semiovale and periventricular regions bilaterally. She was diagnosed as having "senile chorea" with the MRI findings thought to represent iron deposition in the basal ganglia of unknown cause.

Her chorea slowly increased in severity and 5 years after her first assessment reserpine (2 $\mathrm{mg}$ /day) was initiated with moderate improvement in her chorea. Two years later tetrabenazine $(75 \mathrm{mg} /$ day $)$ was substituted with further benefit because of concerns that the reserpine may have been exacerbating her increasing postural instability. She required placement in a nursing home 8 years after the onset of her chorea because of the worsening postural stability and her increasing number of falls. She was last examined at this time and had moderate head rocking movements with mild 


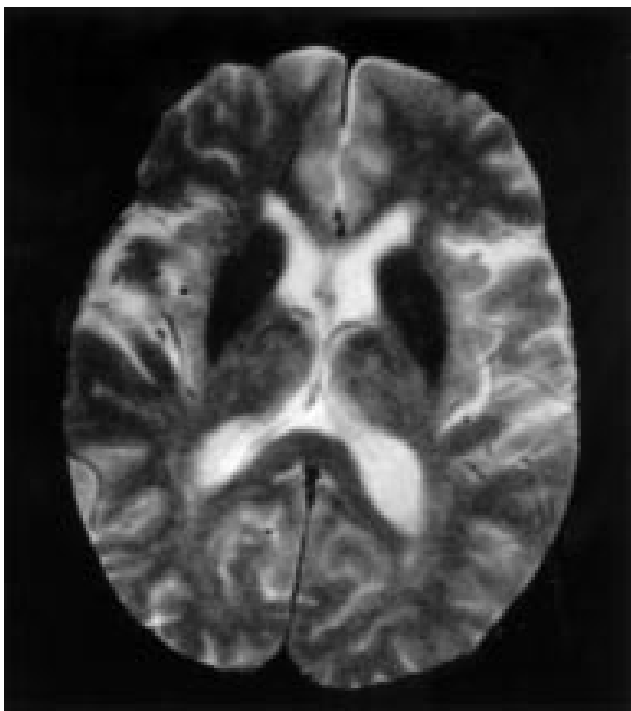

Figure 1 T2 weighted MRI.

generalised chorea that increased with movement. She continued to have only minor memory difficulties. She died suddenly at the age of 85,9 years after the onset of her chorea.
NEUROPATHOLOGICAL FINDINGS

Neuropathological examination was confined to the right half of the brain. External examination of the cerebral convexity showed no significant cortical atrophy but there were two small areas of subarachnoid haemorrhage in the right occipital and superior parietal areas. Coronal sections through the cerebral hemisphere showed a slight flattening of the head of the caudate nucleus. The medial segment of the globus pallidum had a cribriform appearance. The entorhinal cortex was thinner than normal and there was mild atrophy of the amygdala. A small area of cortical infarction was seen underlying the area of subarachnoid haemorrhage in the right superior parietal area. A large haemorrhage measuring $3.5 \times 4.0 \times 8.0 \mathrm{~cm}$ was noted in the occipital lobe extending into the posterior temporal area. Horizontal sections through the brainstem were unremarkable with the substantia nigra and locus ceruleus well pigmented. Sagittal sections through the cerebellum were unremarkable, including the dentate nucleus. Microscopic examination showed severe neuronal loss in the globus pallidus, predominantly in the medial segment, with abundant neuroaxonal spheroids and a moderate degree of iron
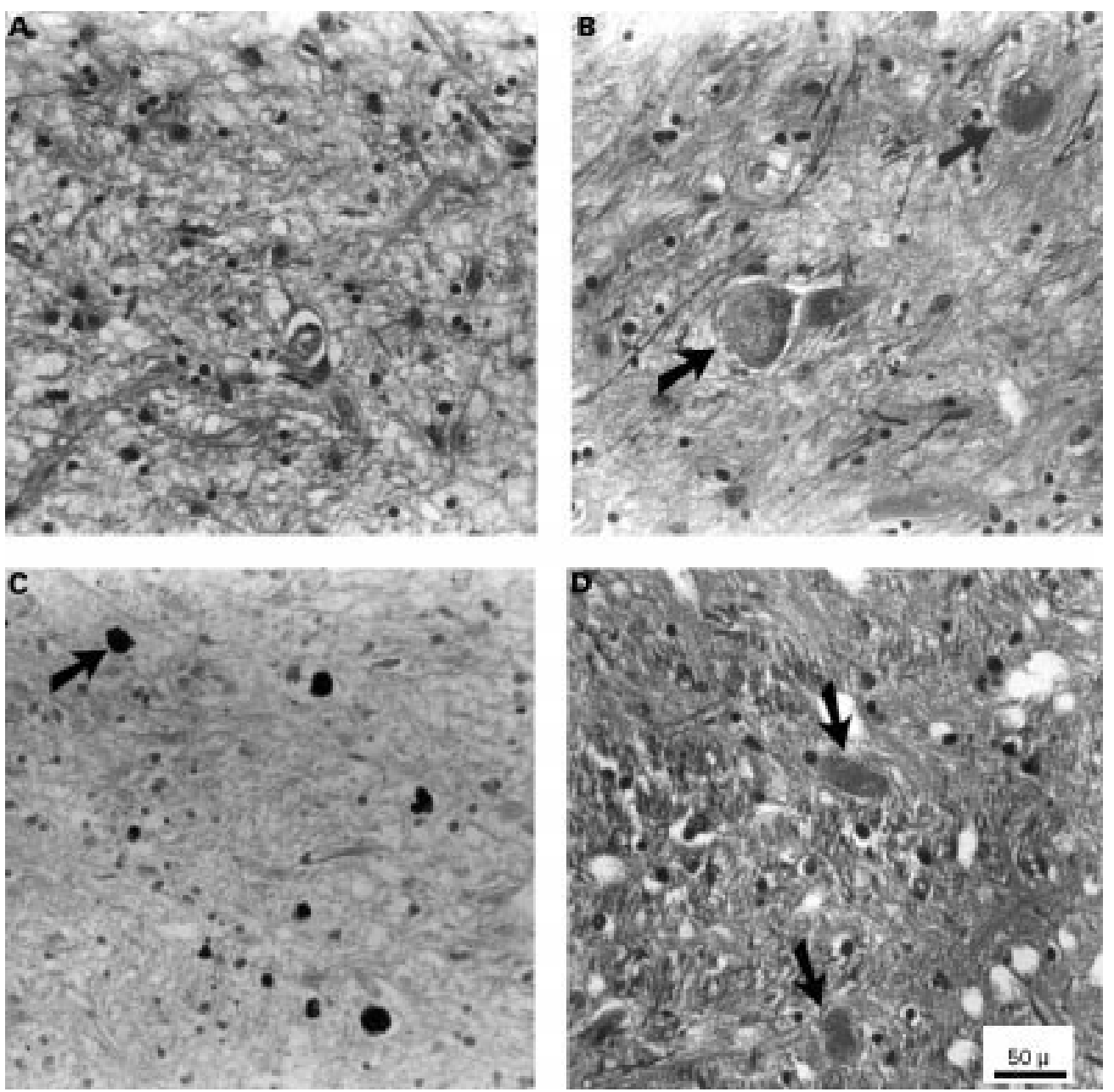

Figure 2 (A) Neuronal loss, (B) spheroids (arrows), and (C) iron pigment deposition (arrow) in the medial segment of the globus pallidus. (D) Spheroids in the substantia nigra, pars reticulata (arrows). Haematoxylin and eosin luxol fast blue $(A, B$, and $D)$. Perl's Prussian blue reaction for iron (C). 
Postmortem cases of late adult onset (age>50 y) Hallervorden-Spatz syndrome

\begin{tabular}{|c|c|c|c|c|}
\hline Study & $\begin{array}{l}\text { Age of } \\
\text { onset }(y)\end{array}$ & $\begin{array}{l}\text { Age of } \\
\text { death }(y)\end{array}$ & Presenting symptoms & Other features \\
\hline Wang et $a l^{14}$ & 63 & 68 & Parkinsonism & Dementia \\
\hline Jankovic et $a \bar{l}$ & 55 & 68 & Parkinsonism & $\begin{array}{l}\text { Blepharospasm, apraxia of eyelid } \\
\text { opening, aphonia, dementia }\end{array}$ \\
\hline Jelinger $e t a l^{15}$ & 64 & 70 & Parkinsonism & Seizures \\
\hline Evrard et $a l^{16}$ & 57 & 62 & Gait difficulty & $\begin{array}{l}\text { Athetosis, facial immobility, } \\
\text { repetitive movements, } \\
\text { contractures, ataxia, dementia, } \\
\text { seizures }\end{array}$ \\
\hline Netsy $e t a l^{17}$ & - & 54 & Unsteady gait & Alcohol, tuberculosis, syphilis \\
\hline Dooling ${ }^{\star}$ et $a l^{1}$ & - & 75 & $\begin{array}{l}\text { Not thought to have } \\
\text { primary neurological } \\
\text { disorder }\end{array}$ & $\begin{array}{l}\text { Chronic alcoholism, generalised } \\
\text { seizures after cerebrovascular } \\
\text { accident }\end{array}$ \\
\hline Dooling ${ }^{\star} e t a l^{1}$ & - & 89 & Dementia & \\
\hline
\end{tabular}

^Limited details given and the patient's specific pathology not described but was part of large postmortem series of Hallervorden-Spatz syndrome

deposition (fig $2 \mathrm{~A}$ and $\mathrm{B}$ ). The iron deposition was most abundant extracellularly and was often perivascular (fig $2 \mathrm{C}$ ). It was also noted in astrocytes and more rarely in neurons. The striatum showed a moderate degree of neuronal loss and gliosis affecting chiefly the large neurons. Iron deposition was also noted, although less abundant than in the globus pallidus and in the same pattern of distribution. Severe neuronal loss was noted in the pars reticulata of the substantia nigra with a moderate degree of iron deposition and neuroaxonal spheroids (fig 2 D). The gracilis and cuneatus nuclei showed large numbers of neuroaxonal spheroids with no significant neuronal loss or pigment deposition. Sections of neocortex showed mild patchy neuronal loss and occasional spheroids. The substantia nigra pars compacta showed no significant neuronal loss and gliosis but a rare spheroid. Mild Alzheimer type changes were present (CERAD: neurofibrillary tangles 0 , neuritic plaques sparse, and diffuse plaques moderate; BRAAK stage II/ VI). In addition to the degenerative changes, there was an old haemorrhagic infarct in the occipital lobe in close proximity to a recent intracerebral haemorrhage. These vascular changes were accompanied by severe amyloid angiopathy.

NEUROPATHOLOGICAL DIAGNOSIS

The neuropathalogical diagnoses were: Hallervorden-Spatz syndrome; old occipital infarction; recent intracerebral haemorrhage due to amyloid angiopathy; mild Alzheimer's changes.

\section{Discussion}

In 1922, Hallervorden and Spatz described the syndrome and pathological findings that characterise the condition that bears their names. ${ }^{6}$ It classically presents as a relentlessly progressive disease of childhood and adolescence in which motor symptoms predominate with prominent rigidity, dystonia, intellectual impairment, and/or pyramidal tract signs. ${ }^{1}$ Choreoathetotic movements or tremor may also be present but these features have not been described in isolation. This "typical" presentation often follows an autosomal recessive pattern of inheritance and has recently been linked to chromosome 20p12.3-p13 in 10 families. ${ }^{7}$ Pathologically, the main features of the disorder consist of (1) neuronal loss and gliosis affecting mainly the internal segment of the globus pallidus and the pars reticulata of the substantia nigra; (2) widely disseminated rounded or oval non-nuclear "spheroids", especially in the globus pallidus and substantia nigra; (3) intracellular and extracellular iron deposition in the above areas. ${ }^{12}$

The pathological findings in our patient are characteristic of Hallervorden-Spatz syndrome including neuronal loss and gliosis, axonal spheroids, and iron deposition in a typical distribution. Iron deposition can be seen in normal aged brains and has been described in other neurological entities, including heredodegenerative and storage diseases. ${ }^{5}$ Axonal spheroids are also found in many conditions and are thought to be the mark of neuroaxonal dystrophies. However, only HallervordenSpatz syndrome is thought to have the unique combination of pallidonigral gliosis, iron deposition, and axonal swellings.

Since Hallervorden and Spatz's original description an increasing number of cases have been reported with "atypical" clinical features such as a cerebellar ataxia, retinitis pigmentosa, and/or a seizure disorder. ${ }^{1}$ Several cases have also been described in adults over the age of 50 (table), often presenting with a progressive dementia or parkinsonism. Neurofibrillary tangles $^{4}$ and Lewy bodies ${ }^{82}$ have also been described in association with the pathology of Hallervorden-Spatz syndrome. This range of clinical features and associated pathologies has encouraged the use of the term HallervordenSpatz "syndrome" instead of "disease". However, it has been proposed that the eponymic designation be dropped completely because of the apparent non-specificity of the pathology and for humanitarian reasons related to their involvement with human experimentation in Nazi Germany. ${ }^{9}$

Senile chorea is an uncommon, apparently sporadic clinical entity, characterised by the presence of a late onset, generalised chorea without dementia. The question of whether it represents a single disease or syndrome had been debated for many years. Some authors had thought that it was due only to late onset Huntington's disease, with death presumably occurring before the mental changes. ${ }^{10}$ As the availability of the trinucleotide CAG genetic testing it has become clear that Huntington's disease may account for up to $50 \%$ of cases, but there is still a subgroup of patients for which no cause is identified despite extensive investigations. ${ }^{11-13}$

The presentation in our patient of isolated chorea is very unusual and has not been associated with Hallervorden-Spatz syndrome pathology. Her premorbid diagnosis was that of "senile chorea", as she had no identifiable cause for her abnormal movements. Huntington's disease was ruled out by her negative CAG genetic testing. Extensive laboratory testing found no abnormalities to suggest an alternative diagnosis. Her MRI did not show the typical "eye of the tiger" sign or pronounced low signal in the globus pallidus without the additional inserted bright signal that has now 
been well documented in patients with Hallervorden-Spatz syndrome. In fact the abnormal low signal, designating excessive iron deposition, was restricted to the striatum despite the greater extent of pallidal involvement found at necropsy. This discrepancy may in part be due to the fact that the MRI was done early in the course of her disease.

It is difficult to explain the wide variability of clinical features that have been associated with the pathology of Hallervorden-Spatz syndrome. It is highly unlikely that our patient's findings arose from the same pathophysiological mechanisms as typical paediatric cases. Hallervorden-Spatz syndrome is an umbrella term for a heterogeneous group of neurodegenerative disorders which will become antiquated as further research improves our understanding and classification.

1 Dooling EC, WC Schoene, EP Richardson Jr. HallervordenSpatz syndrome. Arch Neurol 1974;30:70-83.

2 Halliday W. The nosology of Hallervorden-Spatz disease. $\mathcal{F}$ Neurol Sci 1995;134:84-91.

3 Alberca R, et al. Late onset parkinsonian syndrome in Hallervorden-Spatz disease. 7 Neurol Neurosurg Psychiatry 1987;50:1665-8.

4 Eidelberg D, et al. Adult onset Hallervorden-Spatz disease with neurofibrillary pathology. A discrete clinicopathological entity. Brain 1987;110:993-1013.

5 Jankovic J, et al. Late-onset Hallervorden-Spatz disease presenting as familial parkinsonism. Neurology 1985;35:22734.
6 Hallervordan J, H Spatz. Elgenartige Erkrankung im extrapyramidalen system mit desonderer beteiligung des globus pallidus und der substantia nigra. $Z$ Neuro Psychiat 1922 79:254-302.

7 Taylor TD, Litt M, Kramer P, et al. Homozygosity mapping of Hallervorden-Spatz syndrome to chromosome 20p12.3p13 [published erratum appears in Nat Genet 1997;16:109] Nat Genet 1996;14:479-81.[AQ:2]

8 Tuite J, J Provias, AE Lang. Atypical dopa responsive parkinsonism in a patient with megalencephaly, midbrain Lewy body disease, and some pathological features of Hallervorden-Spatz disease. I Neurol Neurosurg Psychiatry 1996;61:523-7.

9 Harper S. Naming of syndromes and unethical activities: the case of Hallervorden and Spatz [see comments]. Lancet 1996;348:1224-5.

10 Friedman JH, M Ambler. A case of senile chorea. Mov Disord 1990;5:251-3.

11 Garcia Ruiz J, Gomez-Tortosa E, Del Bario A, et al. Senile chorea: a multicenter prospective study. Acta Neurol Scand 1997;95:180-3.

12 Warren JD, Firgaria F, Thompson EM, et al. The causes of sporadic and senile chorea. Aust N Z7 Med 1998;28:42931 .

13 Shinotoh H, et al. Normal CAG repeat length in the Huntington's disease gene in senile chorea. Neurology 1994;44: 2183-4

14 Wang LN, KW Huang, ZY Liu. Sporadic late-onset Hallervorden-Spatz disease presenting as parkinsonism in a Chinese patient. Chin Med f (Engl) 1990;103:686-8.

15 Jellinger K, E Neumayer. Unusual late-onset type of Hallervorden-Spatz disease. Clinico-pathological study of a case presenting as parkinsonism. Z Neurol 1972;203:10518.

16 Evrard E, Hariga J, Martin JJ, et al. Late Hallervorden-Spatz disease with important reticular and cerebellar participation. Psychiatr Neurol Neurochir 1968;71:243-54.

17 Netsky M, D Spiro, H Zimmerman. Hallervorden-Spatz disease and dystonia. I Neuropathol Exp Neurol 1951;10: $125-41$ 